\title{
Video Transformation in Big Video Era and Its Impact on Content Editing
}

\author{
Mingzhi Yin \\ School of Foreign Languages, North China Electric Power University, Beijing, China \\ Email: 1148709315@qq.com
}

How to cite this paper: Yin, M. Z. (2021). Video Transformation in Big Video Era and Its Impact on Content Editing. Open Journal of Social Sciences, 9, 116-124. https://doi.org/10.4236/jss.2021.911010

Received: October 13, 2021

Accepted: November 20, 2021

Published: November 23, 2021

Copyright $\odot 2021$ by author(s) and Scientific Research Publishing Inc. This work is licensed under the Creative Commons Attribution International License (CC BY 4.0).

http://creativecommons.org/licenses/by/4.0/

\begin{abstract}
In the "Big Video Era", the amount of video has increased dramatically and the presentation mode of videos has also changed fundamentally with the development of technology. As huge changes will bring many new opportunities, it is urgent to explore the changes of video in the big video era and its impact on content editing for the development of the video industry. By studying and analyzing the relevant literature, combining the current development of the video industry and specific practical cases, this thesis explores the transformation of video content in the "Big Video Era", analyzes the positive and negative impacts, and discusses the new requirements in video content editing.
\end{abstract}

\section{Keywords}

Big Video Era, Video Transformation, Content Editing

\section{Introduction}

Now we have entered a new era of video, which is the Big Video Era. First of all, what is the Big Video Era? The definition of the Big Video Era is the comprehensive expression of video. In the era of mobile and socialization, there are many different media forms, so it presents the pattern of "big video". The beginning of the Big Video Era is the network video and sharing video, which is also the product of media integration. In the era of big video, technology is being upgraded constantly, and now we have 5G technology.

The Big Video Era has already had a huge impact on video content with the explosion of new videos appearing every day on various platforms and software. John Hoffman, CEO and Director for GSMA Ltd., said at WAIC 2019 that, "We have a huge unexplored frontier, a big opportunity for growth, and unlimited 
potential for growth. Because video is the biggest driver of data flow in the world today, video will bring endless opportunities for all players in the industry" (Embrace the Big Video Era, 2017). Short videos are becoming the mainstream of the day, creating an interceptable, reconfigurable, and embeddable TV stream and video textualization dynamic.

Therefore, in big video era, changes in the way media converge have led to changes in video, which also have a huge impact on content editing. In order to grasp these new opportunities and seize the opportunities created by the times, we need to explore the new requirements for video editing and video content in the big video era in order to better create videos that meet the progress of the times and human aesthetics.

\section{Literature Review}

If you search "big video era" in JSTOR, you can get 5059 results. Of academic content, you can get 424 Journals, 1001 Book Chapters and 576 Research Reports. Of primary source content, you can get 2866 serials, 106 books and 86 documents.

In China, if you search for "big video era" or its related keywords, such as "short video", "big data era", "video content", etc. on CNKI, you can get $2 \mathrm{~W}+$ results. In terms of years, the number of papers shows a gradual increase, and reaches the peak in 2020. Its research content is mainly about short videos, Internet, new media, various short video platforms, media integration, communication strategies, etc.

Its research is mainly based on several directions. Firstly, it is a specific analysis study of a particular short video. Based on a specific short video software to carry out research, to carry out analysis of its data and audience, to carry out case studies. For example, the analysis of Li Ziqi's video in TikTok platform accounts for more than 200 articles. Secondly, it is a kind of research which combines short video and other forms of media together. As an emerging media means, the integration of short video and other media has been attracting much attention, and some researchers are also very interested in this field, and they summarize and explore the integration practice of short video and other media from the current situation of the industry, and summarize their experiences. Articles such as "The road to break the siege of mainstream media in the era of short video" and "How local traditional media can do well in short video to enhance their influence" have been carefully analyzed and studied through the perspective of the mutual influence of short video and traditional media and the drastic impact of short video on traditional media. We can also search a lot of research on the change of traditional media, which is always closely related to the new media and the big video era. When you search for traditional media in the search bar, you will find a large number of papers on traditional media development and innovation. This also coincides with the main tone of this thesis. Changes corresponding to the new media era must occur and new requirements 
must be put forward in order to comply with the new trend. Fourth, the future development trend of short video has also been the mainstream of popular research. For example, Nirobaier Elti and Zheng Liang mentioned in "Characteristics, Trends and Dilemmas of Short Video Content Production in the New Media Era" (Alti \& Liang, 2021) that the content production of short video in the new media era has formed socially driven, emotionally stimulating and multi-valued production modes while developing rapidly, and has shown the integration into more complex In addition. Some scholars have also explored the development of short video from other perspectives, and the prospects for the development of the short video industry from a technological perspective. Some scholars believe that the main development trend of the short video industry should focus on technology upgrading, such as AR, VR, data visualization and other high-tech. These will become an important part of the promotion of short video field updates and iterations.

\section{Analysis of the Positive Impact of the Big Video Era on Video Orientation}

\subsection{The Increase of Public Participation}

The public is involved in the process of video production and grassroots culture is becoming popular. In the past, only authoritative media have the credential to produce and publish videos on public channels. However, nowadays, it is the era of universal video. Around 2010, as media competition intensified and new media, especially online media, cell phones and other electronic media, diverted TV audiences, leading to the decline of TV viewership (Yu, 2021). Thanks to the development of technology, all it takes is a phone for the public to become a video producer. The number of video-based software is also growing. On October 20, 2021, at the launch of Snack Video Short Drama Starmount, the person in charge, $\mathrm{Yu} \mathrm{Ke}$, announced the scale of 230 million daily active users and 770 billion total plays of Snack Video Short Drama (Sun, 2021). The prevalence of short videos on TikTok has made it easier to get started with video production. Everyone can use the function of video shooting, so anyone with a cell phone and WiFi can share their life on the platform. The number of online video releases is growing in spurts. C. Stokel-Walker analyzes the current state of Jitterbug and concludes that Jitterbug has seen a dramatic rise in popularity since its merger with Musical.ly. The users of TikTok open the app an average of 121 times per week and watching nearly 27 minutes per day, showing that the rise of TikTok has a little sign of slowing down (Stokel-Walker, 2020). In this case, the public will prefer videos made by ordinary people to those released by official media, because those videos are more relevant to the subject matter of life and are more interesting.

\subsection{Changes in the Function of Video Discourse}

The discourse function of video has been reinvented. Since video has the func- 
tion of spoken language, it has become our social language. As humans are social-loving animals, they are willing to share those videos which make them have emotional resonance further to their friends or their followers, which further triggers the generation of retweeting behavior. For example, if a cute pet blogger uploads a video of a cat, the cute behavior or beautiful appearance of the cat will make the viewers who like cats or cute things see it and have emotional resonance, thus leading to the generation of retweeting behavior. For example, during the parade of China's National Day, a video called the armed police squad kicking the march blindfolded generated 35,442,000 likes, 109,000 comments and 419,000 retweets due to its passionate soundtrack and content that touched everyone's love for the country (Zhang, 2021a).

\subsection{Reconfiguration of the Video Communication Context and Expansion of the Consumption Scenario}

In the era of big video, the video communication context has been reconstructed, and the consumption scenario has been expanded. The original way and place of video communication may be relatively single, but in the era of big video, the communication context has undergone a radical change. The boom in immersive experiences was brought about by the changing needs of the public at the aesthetic and experiential levels (Huang, 2021a). First, the video itself has become a new communication scenario. With the rise of live video, we can watch the lives of the anchors, getting closer to the scenes of others' lives, and satisfy our own voyeurism. On common video platforms, people are able to send pop-ups, making timely comments on videos, and sharing their own feeling after watching the videos. In addition, some special technologies have been applied to different scenarios, creating many new experiences. For example, in the opening show of League of Legends, the audiences are completely shocked by the exciting sensory experience of the live song performances with VR character interaction and the dazzling VR special effects. As the communication context of video has changed, the science and technology applied to it has further expanded our consumption scenarios. Audition content is closely connected to viewing scenarios, consumption behaviors, and usage contexts. Today we can see audition content entering our lives. When we buy cosmetics such as lipsticks and foundations, we can try them out online and complete the whole shopping behavior without leaving home. For example, Maybelline premiered the world's first AI foundation adapter " $360^{\circ}$ Intelligent Color Selection", using the AI foundation adapter to scan the face and surrounding light environment 360 degrees. Through AI analysis, it can ensure that when consumers buy the new Superstay Makeup Foundation and the classic Fit Me custom water-based foundation online, they can enjoy convenient and intelligent personalized color selection recommendation service to accurately locate the right foundation shade for you.

\subsection{Change in the Form of Video Interaction}

In the era of big video, the interactive form of video is upgraded. In the past, we 
could only sit in front of the TV or computer, watching the characters in TV. There was no way for us to interacte with them. However, interactive narrative, immersive experience, multi-interaction, and other diverse video forms have emerged and come into use nowadays. Artificial intelligence, big data, VR, and other technologies have gradually come into our lives. This has greatly enriched our entertainment life. According to the authoritative industry report $V R$ and $A R$ : Decoding the Next Universal Computing Platform, gaming, live streaming and video entertainment will account for 60 percent of overall VR/AR revenue expectations, and statistics from Goldman Sachs and Sadie Consulting show that the global VR live streaming market will increase in revenue from $\$ 1.161$ billion to $\$ 4.113$ billion from 2021-2025. 4.113 billion (Song, 2021). Cinema has also been updated; IMAX and 4D movies are gradually more and more common, ensuring us to have a better screen viewing experience. Those cool future times in technology movies seem to be no longer far away from us. Google Glass has shone a ray of success into reality. It combined AR, VR with the glasses, equipped them with a variety of intelligent functions, so that the flattened glasses can bring a brand new audition experience. Although this technology is still immature, I believe that human beings will be able to make a breakthrough in this technology with the fast-developing technology.

\section{Analysis of the Negative Impact of the Big Video Era on Video Orientation}

\subsection{Lack of Control over Error Messages}

Due to the technological development in the era of big video, the information flow is accelerated. While the efficiency of the delivery of correct information is improved, wrong information is also being delivered faster. Many media, as well as marketing numbers, often publish misguided statements in order to gain attention, inciting public sentiment and misleading audiences, resulting in bad consequences. Although many platforms have increased the number of machine and manual audits, we still haven't found a completely effective way to thoroughly screen the authenticity of information today. In order to prevent public riot, confusion, and panic caused by wrong information, it is extremely essential not only to improve the quality of video and content output from ourselves, but also to generate video screening means that match with the Big Video Era.

\subsection{The Serious Problem of Video Quality and Content Homogenization}

The diversification of the media and the simplification of blogger entrance has resulted the video quality to vary. Some data show that more than $30 \%$ of consumer users believe that there is too much homogenized content in TikTok, and the phenomenon of following the trend of shooting has led to a decrease in goodwill of some users, resulting in the loss of a large number of short video users (Deng, 2020). In order to be popular, some bloggers may be unscrupulous 
and choose to copy other people's videos. However, for now, there is neither a very effective mechanism to determine whether a video is plagiarized, nor strict penalties to punish plagiarism, which has allowed plagiarism to run even more rampant. The 2020 China Internet Short Video Copyright Monitoring Report released by 12,426 Copyright Monitoring Center shows that between 2019 and October 202, a total of 16,026,900 suspected infringement links were monitored, and the rate of exclusive original creators being infringed was as high as $92.9 \%$ (Zhang, 2021b). This is a great disrespect to the hard-working original video makers. As a positive example, YouTube has stricter requirements on whether a video is original or not, even the use of background music without consent is considered as a severe violation. When multiple unoriginal acts are found, the account of this video producer will be directly banned forever, which greatly reduces the number of plagiarism. In the past, the delivery of information was point-to-point and the media was able to control the quality of video content as much as possible. But considering the universal use of video software today, it is difficult for us to make strict requirements for the quality of the video, which has led to the quality issue of the video, and even some vulgar videos and videos with incorrect values are still being delivered in the network. Improving the quality of original user content is imminent. In The Research of Short Video App: The Case of TikTok, Janssen Richardson examines the factors that make TikTok short videos successful in terms of platform marketing strategy and user use and satisfaction theory, and argues that in a short video space with intense content homogenization, only content of sufficient quality can make In the short video space with intense content homogeneity, only high quality content can lead to higher user survival rate (Richardson, 2019). In the Big Video Era, in order to better restrain video content and distribution channels, further laws and a better system are required urgently.

\subsection{Lack of Guidance on Correct Value Orientation for Teenagers}

In the Big Video Era, children are exposed to the Internet at an early age. Until June 2020, the teenage group aged 10 to 19 years old accounted for $14.8 \%$ of the short video users. Students have a high share of $23.7 \%$ in the occupational dimension (Huang, 2021b). However, some of the videos on the Internet don't have good qualities, and some of them may be misleading in their translated values. Some of the videos are made by people who overemphasize the role of love in a person's life, or who are overly interested in money and material life. These videos along with the bad Internet environment will have a side effect for teenagers who are at the age of forming their own view towards the world. Nowadays, we have started to pay attention to applying stricter control of video quality, but Rome is not built in one day. Current major video platforms have launched a youth model that can effectively block some of the videos with improper values, but still cannot solve the problem fundamentally. Relevant policies and programs should be introduced as soon as possible to positively guide 
young people searching the Internet and viewing online videos, growing up healthily in the Big Video Era.

\section{New Requirements of Video Content Editing in the Big Video Era}

\subsection{To Tell Good Stories}

Video editors need to be able to tell good stories. With the explosion of video information in the Big Video Era and the accelerated pace of people's lives, people usually have only fragmented time. As a result, their demand for stories has become higher. Many stories today lack vividness and a sense of immersion, and are just flat and straightforward, so we need to help the public distill effective information and tell the best stories in the shortest time. Nowadays, a short video format for narrating stories is quietly gaining popularity on various platforms. As people don't have time to watch a whole TV series by themselves, this narrated form of short video will edit a good TV series, leaving the best bits and presenting them to the audience with a narration. The editing in this way is a case of telling a good story. Only those quality contents will be recognized by the public. Creating a good story from this requires more of a blend of various elements and eventually create work with soul.

\subsection{To Tell Right Stories}

The story after video editing should have been positive in its nature. Nowadays, people are enjoying various ways of entertainment, TV and online entertainment programs are no longer the only way for people to find fun. So some programs do whatever they can for the sake of attracting followers, using editing to constantly intensify conflicts and triggering online exposure of someone or something to contribute to the explosion of the program. This is not something we want. When editing, we should pursue more high-quality content output and try to establish the guidance of the right values,, rather than deliberately create intensified conflicts.

\subsection{To Tell Creative Stories}

As mentioned in the previous article, the era of big video is full of opportunities. I think whoever has more creativity and innovation is blessed with bigger chance of success. Originality and uniqueness of content are advocated by the cultural and creative industry and the media industry (Zhao, 2021). The core of the "traffic code" is creativity. As today's videos are mostly the same, people can reap a better response if the output of the video can be creative. On the short video platform, there is always creative editorial content output. For example, launching new challenges, using filters and face effects to shoot videos, technical streaming videos, jamming videos, including the recent use of artificial intelligence effects to complete the video, and so on. All these ways combine music, special effects, VR, and other elements with video in a good way, bringing people 
a new audio-visual experience.

Creativity is important not only for content editing in short videos, but is also indispensable for long videos. The Korean variety show "Heart Signal" series exploded all over the internet because it is a very creative reality show. With a new theme, excellent characterization, and perfect music, it created a brand new relationship variety show. The content explored and the events that really happened in the show also resonated with the audience.

\section{Conclusion}

After studying the two aspects above in general, we can finally give out a conclusion. Big video era has put forward different requirements for video. Firstly, we should combine some of the existing advantages of the big video era, make full use of audience participation, leverage the dividends of multiple platforms, develop the technologies of $\mathrm{AR}, \mathrm{VR}$ and further combine these technologies with the video industry. At the same time, we should also further improve the existing loopholes and introduce relevant regulations and laws to regulate various negative behaviors on the Internet as soon as possible, so as to create a healthy and positive network platform for the teenagers and let video communication become the main body of information delivery. In the process of video content output, we also need to combine the new requirements of the big video era and pay further attention to the content of video. While outputting high-quality original internal stories, we should also focus on its form. We have to add innovative content and ideas to the content so as to further attract the attention of all the viewers.

"Let content creation present maximum value", says the editor of the Audiovisual Department. In contrast to equal opportunities for content producers, users have a very limited attention span. “As a result, users' choices become quite valuable. In this case, quality content can stand out and realize its headline value. Vertical and distinctive niche and niche programs can also reap their segmentation value and even long-tail value from them" (Zhao, 2018).

In the Big Video Era, the transformation of videos will undoubtedly be a new challenge for all media people, and video-based communication innovation has infinite possibilities. For us to create works that are valuable, worth watching, and worth repeating, we still need further in-depth learning, thinking, and practice!

\section{Conflicts of Interest}

The author declares no conflicts of interest regarding the publication of this paper.

\section{References}

Alti, N. \& Liang, Z. (2021). Characteristics, Trends and Dilemmas of Short Video Content Production in the New Media Era. China Editorial, No. 3, 81-85. 
Deng, Y.-S. (2020). Research on the Communication Development Strategy of Jitterbug Short Video in the Context of the Internet. Journalism and Culture Construction, No. 17, 167-168.

Embrace the Big Video Era (2017). https://www.huawei.com/cn/events/ubbf2017/big-video-era

Huang, C. (2021a). Analysis of the Application of Artificial Intelligence in Multimedia Art. Art Pinnacle, No. 27, 129-130.

Huang, W. W. (2021b). The Influence of Jitterbug on Teenagers and Guidance Strategies. Journalism Research Guide, No. 6, 79-80.

Richardson, J. (2019). The Research of Short Video App: The Case of TikTok. IEEE Transactions on Multimudha, 3, 91-100.

Song, J. (2021). VR Live Streaming: 5G Helps Usher in the Market Inflection Point. China Electronics News, No. 4, p. 2.

Stokel-Walker, C. (2020). Tik Tok's Global Surge. New Scientist, No. 245, 31.

Sun, L. (2021). Racer Sunshine Short Drama Business Report Card: 850 Short Dramas Played over 100 Million. Chongqing Business Daily, No. 4, p. 1.

Yu, W. D. (2021). A Review of Twenty Years of Viewership Application in Shanghai. Shanghai Radio and Television Research, No. 2, 114-121.

Zhang, C.-C. (2021b). Short Video Clips: A Strong Demand That Can't Be Suppressed in the Legal Crevice. Youth Journalist, No. 9, 111.

Zhang, Y. L. (2021a). Short Video Communication and National Identity Construction of the 70th Anniversary National Day Parade. Master's Thesis, Lanzhou: Lanzhou University.

Zhao, G. H.. (2021). Research on Short Video Production and Dissemination Mechanism in the Era of Big Data. Media, 13, 50-52.

Zhao, Q. J. (2018). Good Programming in the Era of Big Video. Audiovisual World, No. 1, p. 1 . 